\title{
Pneumomédiastin en chantant
}

\section{Pneumomediastinum Singing}

\section{E. Hansconrad}

Reçu le 19 juin 2015 ; accepté le 16 juillet 2015

(C) SFMU et Lavoisier SAS 2015
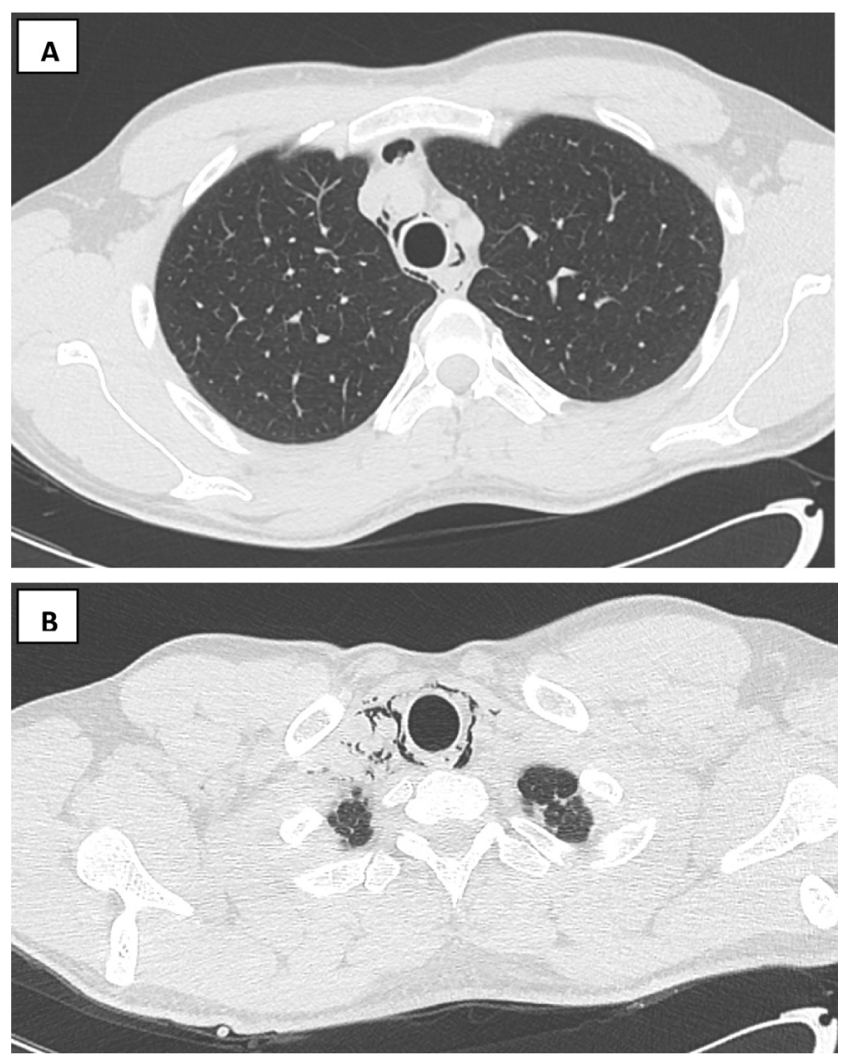

Fig. 1 Tomodensitométrie cervico-thoracique en fenêtre parenchymateuse (A et B) montrant le pneumomédiastin avec l'emphysème des espaces cervicaux profond
Un homme de 29 ans, sans antécédents, chanteur de profession, consulte aux urgences pour une douleur médiothoracique et cervicale intense, nécessitant une antalgie par morphiniques, à type de brûlure ascendante, augmentée à la déglutition et à l'inspiration, apparue brutalement il y a 24 heures pendant qu'il chantait. Devant cette douleur thoracique atypique, intense et inexpliquée par la normalité de l'examen clinique, de l'électrocardiogramme, de la biologie et de la radio de thorax, une tomodensitométrie cervicothoracique a été réalisée et retrouvait un pneumomédiastin avec un emphysème des espaces cervicaux profonds (Fig. 1). Une consultation avec un otorhinolaryngologiste (ORL) et la réalisation d'une nasofibroscopie ne retrouvait pas de brèche identifiée permettant au patient de rentrer à domicile avec une contre-indication aux efforts, des antalgiques et une consultation ORL de réévaluation dans 15 jours. Un pneumomédiastin est une pathologie potentiellement grave ; il est la conséquence d'un barotraumatisme causant la rupture d'une bronchiole terminale. Les circonstances de l'élévation de la pression intra-alvéolaire sont variables et toute douleur thoracique aux urgences faisant suite à un effort [1], même paraissant bénin, doit faire suspecter cette pathologie et une tomodensitométrie cervicothoracique doit être réalisée si besoin.

\section{Références}

1. Shine NP, Lacy P, Conlon B, McShane D (2005) Spontaneous retropharyngeal and cervical emphysema: a rare singer's injury. Ear Nose Throat J 84:726-7 